\title{
Technological characterization of Lactobacillus in semihard artisanal goat cheeses from different Mediterranean areas for potential use as nonstarter lactic acid bacteria
}

\author{
Zhaoxu Meng, ${ }^{*}$ Lanwei Zhang, ${ }^{*} \dagger^{1}$ Liang Xin, ${ }^{*}$ Kai Lin, ${ }^{*}$ HuaXi Yi,,${ }^{1}$ and Xue Han* \\ *School of Chemistry and Chemical Engineering, Harbin Institute of Technology, Harbin 150000, China \\ †College of Food Science and Engineering, Ocean University of China, Qingdao 266003, China
}

\section{ABSTRACT}

The potential of 25 Lactobacillus isolates from 8 semihard artisanal goat cheeses manufactured in 4 different Mediterranean areas was examined for use as nonstarter lactic acid bacteria. The isolates were identified using $16 \mathrm{~S}$ rDNA sequence analysis. Sixteen strains belonged to Lactobacillus paracasei and 9 to Lactobacillus rhamnosus. The isolates were first screened for salt tolerance, exopolysaccharide and diacetyl production, proteolytic and lipolytic activity, and acidification and autolyzing capacities. Most of the lactobacilli displayed strong salt tolerance [20 strains, including 13 of $L b$. paracasei and 7 of Lb. rhamnosus, could grow at $6 \%$ (wt/vol) salt], low acidification activity (16 strains, including 9 of $L b$. paracasei and 7 of Lb. rhamnosus, presented change in $\mathrm{pH} \leq 0.4 \mathrm{U}$ after $6 \mathrm{~h}$ of growth), and high autolytic activity (14 strains, including 9 of $L b$. paracasei and 5 of $L b$. rhamnosus, showed autolysis values ranging between 25 and $65 \%$ ). Eleven $L b$. paracasei and $6 L$ L . rhamnosus produced exopolysaccharide, whereas $8 \mathrm{Lb}$. paracasei and $4 \mathrm{Lb}$. rhamnosus produced diacetyl. Moreover, $9 \mathrm{Lb}$. paracasei and $6 \mathrm{Lb}$. rhamnosus showed proteolytic activity; none of the isolates showed lipolytic activity. Based on the above characteristics, 8 strains were further evaluated for peptidase activity, including aminopeptidase, dipeptidyl aminopeptidase, and dipeptidase activities. The results indicated that all strains showed peptidase activity toward selected substrates. The substrate specificity and extent of peptidase activities were strain-dependent. Four strains (A-3, B-4, D-3, and D-8) presented the best characteristics and represented the most promising nonstarter lactic acid bacteria candidates for use in industrial manufacturing of goat cheese.

Key words: goat cheese, Lactobacillus, NSLAB

Received October 17, 2017.

Accepted December 18, 2017.

${ }^{1}$ Corresponding authors: zhanglw@hit.edu.cn and yihx@ouc.edu.cn
INTRODUCTION

Goat milk distinguishes itself from cow milk by higher digestibility, distinct alkalinity, higher buffering capacity, and presence of certain medicinal and therapeutic values (de Almeida Júnior et al., 2015); therefore, the production and fabrication of goat cheese has shown a growing trend in recent years. Cheese, a dairy product, has been part of the human diet for centuries and greatly affects human nutrition. Artisanal cheeses are manufactured in farmhouses following traditional techniques without the deliberate addition of selected starter cultures. They are typically characterized by a unique taste due to the spontaneous fermentation of unpasteurized milk and are greatly appreciated by consumers around the world. Reports show that their organoleptic characteristics correlate strongly with nutritional characteristics and the environmental contamination level of milk used for cheese production, the manufacturing process, and the presence of appropriate lactic acid bacteria (LAB) for fermentation (Carafa et al., 2015). In general, cheese production comprises 2 different microbiological steps, in which different LAB are involved. The first step, namely the manufacturing of cheese, requires starter LAB; the second step, ripening of the cheese, takes advantages of secondary microbiota nonstarter LAB (NSLAB; Di Grigoli et al., 2015), which are typically used in raw milk cheeses (Carafa et al., 2015). Fermentation properties of the NSLAB strongly affect the sensorial characteristics of the finished cheese.

The family of NSLAB consists of mostly of facultative heterofermentative mesophilic lactobacilli species such as Lactobacillus casei, Lactobacillus paracasei, Lactobacillus rhamnosus, and Lactobacillus plantarum. It also contains of other genera such as Pediococcus, Leuconostoc, and Micrococcus (Franciosi et al., 2009; Piras et al., 2013; Bozoudi et al., 2016). Typically NSLAB grow after cheese brining, as they are resistant to heat and acid treatment that occur during cheese manufacturing and maturation. Bacterial autolysis pro- 
vokes the release of enzymes during the final months of a long maturation process (Martínez et al., 2011; Lazzi et al., 2016). Although industrial cheese production is now well established, the NSLAB composition remains an uncontrolled factor and is suspected to be the cause of inconsistent quality of hard and semihard cheeses (Briggiler-Marcó et al., 2007; Burns et al., 2012). In fact, reports show that alterations in the dominant strains of NSLAB can lead to the formation of off-flavors and biogenic amines in addition to the possibility of gas blowing (Di Cagno et al., 2012). Therefore, to ensure consistency in cheese manufacturing and improve its sensory properties, potent NSLAB strains should be carefully selected when used as adjuncts for controlling the adventitious growth of undesirable NSLAB.

Mediterranean countries are producers of goat milk that is mostly used for cheese production (Juan et al., 2016). Indeed, traditional dairy products derived from goat milk represent a viable sector in the national economy of many Mediterranean countries, such as Spain, Greece, and Turkey, among others (Navarro-Alarcón et al., 2011; Pacheco Da Silva et al., 2016). In most Mediterranean countries, cheeses of raw goat milk represent a significant proportion of ripened cheeses (Serhan et al., 2010).

The most promising bacteria for adjunct cultures are those isolated from indigenous microflora of traditional products. In the present study, we isolated and characterized LAB from artisanal semihard goat cheeses of different Mediterranean countries for developing specific NSLAB. We then evaluated salt tolerance, acidification, autolytic activity, and enzymatic activity of the NSLAB.

\section{MATERIALS AND METHODS}

\section{Cheese Samples}

Eight semihard raw goat milk cheeses were purchased from local stores of 4 Mediterranean areas, namely, Ibores (sample A), Tenerife (sample B), and Babia (sample C) from Spain, Batzos (sample D) and Xinotyri (sample E) from Greece, Sepet (sample F) and Tulumn (sample G) from Turkey, and Darfiyeh (sample H) from Lebanon.

\section{Microbiological Analysis}

To obtain abundant NSLAB from these cheeses, 2 portions (about $10 \mathrm{~g}$ ) per sample were collected using a sterile knife, specifically from the rind (sampled from the first $5 \mathrm{~mm}$ on each side of the cheese, including the edges) and the core (sampled from the center of cheese). The samples were then mixed for continuous microbiological analysis. Cheese samples were homogenized following the procedure described by Tsafrakidou et al. (2016). Briefly, cheese samples were homogenized with $90 \mathrm{~mL}$ of sterile $20 \mathrm{~g} / \mathrm{L}$ sodium citrate solution at $45^{\circ} \mathrm{C}$ in a Stomacher 400 laboratory blender (Seward, London, UK) for 4 min at maximum speed. Dilutions $(1 / 10)$ of the homogenates were prepared with a sterile solution of $0.85 \%$ (wt/vol) sodium chloride and then plated on de Man, Rogosa, and Sharpe (MRS; pH 5.7) agar plates that were incubated under anaerobic conditions at $30^{\circ} \mathrm{C}$ for $5 \mathrm{~d}$ before presumptive lactobacilli examination. Fifteen colonies per sample were randomly picked from the MRS agar plates, totaling to 120 colonies. Twenty-five colonies, which were gram-positive, catalase-negative, and able to grow at 15 and $45^{\circ} \mathrm{C}$, were selected for the next test. Finally, pure cultures were frozen $\left(-80^{\circ} \mathrm{C}\right)$ in MRS broth containing $20 \%$ (vol/vol) glycerol for storage. Isolates were activated by successive transfer in their respective medium and incubation at $37^{\circ} \mathrm{C}$ for production.

\section{Identification of Isolates}

Isolates were identified using the $16 \mathrm{~S}$ rDNA sequencing method. Briefly, genomic DNA was extracted using the E.Z.N.A. bacterial DNA kit (Omega Bio-Tek, Norcross, GA) and amplified by the $16 \mathrm{~S}$ rDNA universal pair of primers 27F: 5'-AGAGTTTGATCCTGGCTCAG-3', and 1492R: 5'-TACGGTTACCTTGTTACGACTT-3'. The amplified products were sequenced by Sangon Biotech Co. Ltd. (Shanghai, China). Sequence similarity searches were performed by comparing the isolated sequences with the ones collected in the GenBank using the BLAST search program of the National Centre for Biotechnology Information (http://www .ncbi.nlm.nih.gov).

\section{Technological Characterization}

Salt Tolerance. The ability of the strains to grow in increasing saline solutions of 2, 6, and 10\% (wt/vol) $\mathrm{NaCl}$ was evaluated according to the method described by Ferrari et al. (2016). After incubation at $37^{\circ} \mathrm{C}$ for 48 $\mathrm{h}$, a color change of the culture from purple to yellow due to acidification of the substrates was considered as positive growth.

Exopolysaccharide and Diacetyl Production. For screening of exopolysaccharide (EPS) production, strains were grown in modified MRS agar medium in which the glucose present in the original formulation was replaced by $10 \%$ sucrose (Fluka, Buchs, Switzerland). Cultures were then streaked on plates that were 
incubated at $37^{\circ} \mathrm{C}$. After incubation for $72 \mathrm{~h}$, possible EPS production was assessed based on the presence of a mucoid or ropy colony using the loop touch test described by Fguiri et al. (2016). Diacetyl production was visually determined by distinguishing a red ring at the top of the culture tubes as described by Ribeiro et al. (2014).

Acidification and Autolytic Activity. The revitalized strains were subcultured in sterile reconstituted goat milk (100 g/L, Meiling, Shanxi, China) to determine the acidification activity. The $\mathrm{pH}$ changes $(\Delta \mathbf{p H})$ were measured after $6 \mathrm{~h}$ of incubation at $30^{\circ} \mathrm{C}$ with a pH meter (PB-10, Sartorius, Goettingen, Germany).

Autolysis was determined on cells prepared according to the method described by Scatassa et al. (2015). The autolytic capacity of the resuspended pellets was measured after $24 \mathrm{~h}$ of inoculation.

Proteolytic and Lipolytic Activities. The proteolytic activity was determined according to the procedure described by de Almeida Júnior et al. (2015). Briefly, strains were plated on the surface of skim milk agar, prepared by supplementing the plate count agar medium with $1 \%$ (wt/vol) skim milk powder. Subsequently, the strains were incubated at $37^{\circ} \mathrm{C}$ for $48 \mathrm{~h}$. The transparent halo-forming colonies were considered positive for proteolytic activity.

The lipolytic activity was tested by cultivating strains on MRS agar with $0.1 \%$ tributyrin at $37^{\circ} \mathrm{C}$ for $72 \mathrm{~h}$. Lipolytic activity was detected by clear zones against a turbid background of emulsified, nonhydrolyzed lipids on the tributyrin medium.

Aminopeptidase, Dipeptidyl Aminopeptidase, and Dipeptidase Activities. All the chemicals used in the assays were purchased from Sigma-Aldrich (St. Louis, MO) and were of reagent or higher grade. The aminopeptidase activity of the selected strains was assayed using Lys- $\rho$-nitroanilide $(\boldsymbol{\rho N A})$, Glu- $\rho \mathrm{NA}$, and Pro- $\rho$ NA as substrates, as described by González et al. (2010). One unit of aminopeptidase activity was defined as the amount of enzyme triggering an absorbance increase of 0.001 unit. Aminopeptidase activity was expressed as the number of activity units per milligram of protein per minute. Dipeptidyl aminopeptidase activity was determined using Gly-Pro- $\rho$ NA and Arg-Pro- $\rho \mathrm{NA}$ as substrates, in accordance with the procedure described by Jensen and Ardö (2010). The activities were expressed as nanomoles of nitroaniline released per minute and per milligram of protein.

The dipeptidase activity was tested on the substrates Leu-Leu, Gly-Glu, and Leu-Pro according to the method described by González et al. (2010). The concentrations of AA released by hydrolysis were estimated by measuring the absorbance at $507 \mathrm{~nm}$. One unit of activity was defined as the amount of enzyme that produced an increase in the absorbance signal at $507 \mathrm{~nm}$ of 0.1 unit in $1 \mathrm{~min}$.

\section{Statistical Analysis}

Each strain was tested for growth in the presence of different $\mathrm{NaCl}$ concentrations in duplicate and in triplicate for the other activity tests. A one-way ANOVA analysis was applied to the results obtained from the activities, using the Student-Newman-Keuls test for comparison of the mean values $(P<0.05)$. The SPSS software package (version 16.0, SPSS Inc., Chicago, IL) was used for this purpose.

\section{RESULTS AND DISCUSSION}

\section{Identification of Lactobacilli}

Twenty-five isolates were obtained from 8 artisanal semihard goat cheeses collected from different Mediterranean regions (Table 1). These isolates were initially identified as lactobacilli based on the alignment success rate with the $16 \mathrm{~S}$ rDNA sequence. Among them, 16 (2 from Ibore, 1 from Tenerife, 5 from Babia, 3 from Batzos, 2 from Xinotyri, 1 from Tulumn, and 2 from Darfiyeh) were identified as $L b$. paracase $i$ and 9 (1 from Ibore, 1 from Tenerife, 1 from Babia, 1 from Batzos, 4 from Sepet, and 1 from Tulumn) were categorized as $L b$. rhamnosus. Strains of the $L b$. casei group, notably either Lb. paracasei or Lb. rhamnosus, occur in cheese mainly as added adjunct cultures (Gobbetti and Minervini, 2014); these strains are also promising candidates for probiotic use. Interestingly, both species were previously isolated from raw goat milk cheeses (Sánchez et al., 2005; Martín-Platero et al., 2009). Lactobacillus paracase $i$ was also isolated from goat milk of extensively small-scale dairy farms in Brazil (de Almeida Júnior et al., 2015). Moreover, Lb. paracasei was also isolated from cheeses Tenerife and Batzos (Hernández et al., 2005; Litopoulou-Tzanetaki and Tzanetakis, 2011). However, Lb. paracasei and Lb. rhamnosus were not isolated from Sepet cheese in the previous study (Ercan et al., 2014), possibly because of differences in sampling season. Surprisingly, the studied cheeses showed distinctive organoleptic profiles, although the core microbiota of the NSLAB belonged to the same species, namely, $L b$. paracase $i$ and $L b$. rhamnosus. This could be because the isolated strains possess specific physiological and technological characteristics that render unique taste to these traditional products, although they belong to species commonly used for the ripening process (Bonomo and Salzano, 2013). Our results are in accordance with 
those reported by several previous studies, highlighting that $L b$. paracasei and $L b$. rhamnosus form the core microbiota of the NSLAB population (Fröhlich-Wyder et al., 2013; Gatti et al., 2014). This is probably due to their high tolerance toward the hostile conditions of the cheese environment during ripening, such as low $\mathrm{pH}$ (4.9-5.3), high salt content (4.0-6.0\%), and the lack of fermentable carbohydrate (Gobbetti et al., 2015).

\section{Salt Tolerance}

Salt tolerance is an important characteristic because NSLAB are exposed to high salt concentrations during cheese manufacturing, notably during the ripening phase (Terzic-Vidojevic et al., 2014; Carafa et al., 2015). Sodium chloride tolerance tests revealed that all strains were able to grow in the presence of $2 \%$ (wt/ vol) salt (Table 1). Moreover, the results indicated that 20 strains (13 Lb. paracasei and 7 Lb. rhamnosus) could grow in the presence of $6 \%$ (wt/vol) salt. Interestingly, 10 isolates (6 Lb. paracasei and $4 \mathrm{Lb}$. rhamnosus) were able to grow in the presence of $10 \%$ (wt/vol) salt. All the 4 isolates of Sepet cheese (F-3, F-22, F-23, and F-25) were able to grow in a buffer containing $10 \%$ (wt/ vol) salt, which may be related to the high salt content $(14 \% \mathrm{NaCl})$ of the Sepet cheese. Salt tolerance $(8 \% \mathrm{wt} /$ vol salt) of $L b$. paracasei and $L b$. rhamnosus isolated from spontaneously fermented mountain cheese were previously reported by Carafa et al. (2015). Lactic acid bacteria uses various mechanisms for salt tolerance, such as the uptake or synthesis of a limited number of solutes (de Almeida Júnior et al., 2015). Sodium chloride is an essential ingredient used in the food industry to boost the sensory characteristics of products and to satisfy the recommended human daily intake of $\mathrm{NaCl}$. Additionally, $\mathrm{NaCl}$ is widely used as a preservative for long storage cheeses and is important for controlling cheese ripening (Georgieva et al., 2009).

\section{EPS and Diacetyl Production}

Lactic acid bacteria produce extracellular sugar polymers called EPS during bacterial growth, which can improve the texture and viscosity of the final product (Fguiri et al., 2016). Colonies exhibiting a slimy appearance were considered positive of EPS production. Among the isolated strains from the collected goat cheese samples, $11 \mathrm{Lb}$. paracasei and $6 \mathrm{Lb}$. rhamnosus strains were observed to produce EPS (Table 1). Corroborating our results, Ferrari et al. (2016) and Ayad et al. (2004) reported that Lb. paracasei and Lb. rhamnosus isolated from goat dairies and dairy products also produced EPS.
The ability to generate EPS is considered as an important feature for LAB used in dairy products, as smooth and creamy products are more appealing to consumers; thus, EPS production is a key feature to be considered for selecting NSLAB (Franciosi et al., 2009). In addition, EPS contributes to the water retention capacity that reduces the calorific content of the end product (Ferrari et al., 2016). From a health standpoint, EPS production by LAB received increased attention due to its immunogenic properties (Domingos-Lopes et al., 2017). Indeed, adhesion to human intestinal mucus and LAB biofilm formation are also mediated by EPS and are related to cholesterol lowering, immunomodulation, and antitumorogenic and prebiotic effects (Guidone et al., 2014). For higher production of EPS, the medium must be supplemented with considerable amounts of a carbon source; additionally, temperature and $\mathrm{pH}$ can affect EPS production (Ferrari et al., 2016). However, the use of EPS in the dairy industry is limited by the low yield of the product, an issue that currently remains unresolved (Ferrari et al., 2016).

In fermented food products, such as cheese, distinct flavors are generated from the microbial production of aromatic compounds. For instance, diacetyl is an essential component of many dairy products, and even at low concentrations it provides a typical flavor and a buttery aroma of cheese (Ferrari et al., 2016). Diacetyl, derived from citrate metabolism, possesses inhibitory activity against food-borne pathogens and is not present in all types of LAB (Thierry et al., 2015). In our study, more than half of the total isolates $(56 \%)$ produced diacetyl. The results indicated that $53 \%$ of the $L b$. paracasei and $67 \%$ of $L b$. rhamnosus could produce diacetyl (Table 1 ). Similar results were reported for lactobacilli from Grana cheese (Monfredini et al., 2012). In agreement with our results, Nikolic et al. (2008) also observed that a high proportion of $L b$. paracasei isolated from goat cheeses have the ability to produce diacetyl. Pérez et al. (2003) observed that $95.2 \%(20 / 21)$ of $L b$. paracasei from Tenerife cheese were able to produce diacetyl, whereas $100 \%(2 / 2)$ of $L b$. paracasei from Tenerife cheese in our study were able to produce diacetyl. de Almeida Júnior et al. (2015) observed that all LAB from raw goat milk samples were weak diacetyl producers.

\section{Acidification and Autolytic Activity}

The results of the acidification activity recorded after $6 \mathrm{~h}$ of bacterial growth in skim milk are shown in Figure 1a. After $6 \mathrm{~h}$, the isolates presented values of $\Delta \mathrm{pH}$ ranging from 0.15 to $0.63 \mathrm{pH}$ units. The results supported the values recorded for lactobacilli isolated from Armada goat cheese by Herreros et al. (2003). 


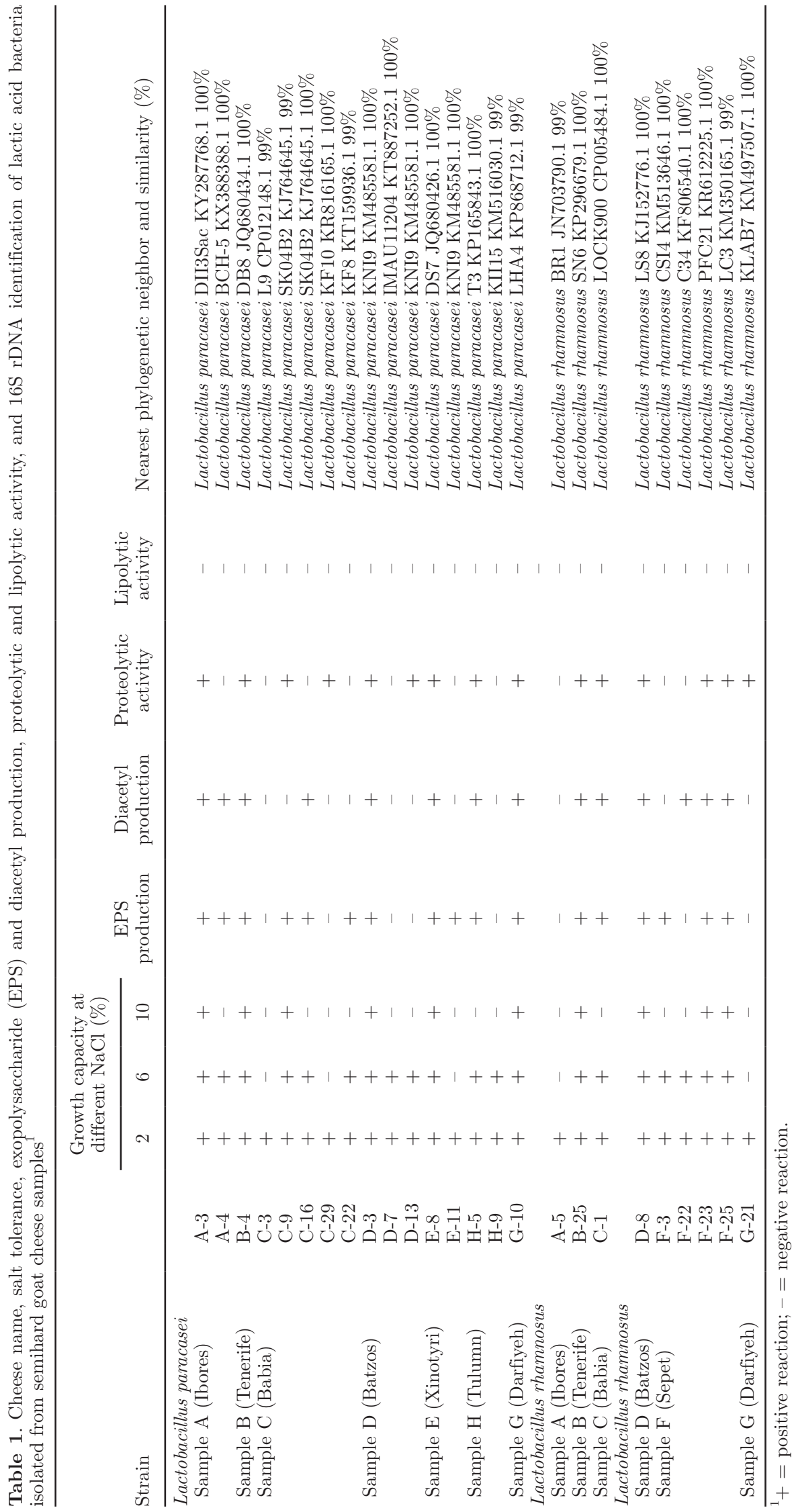


a)

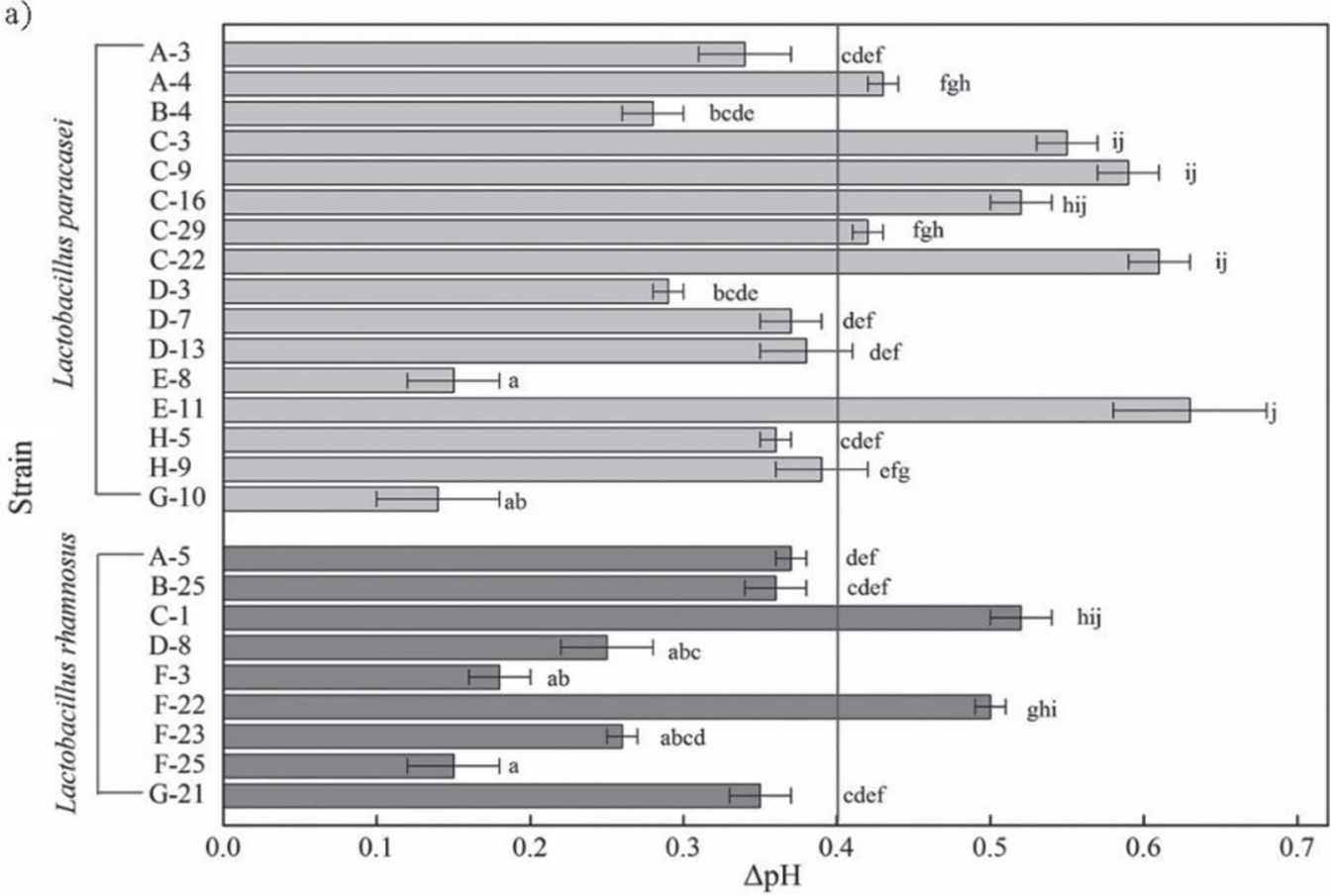

b)

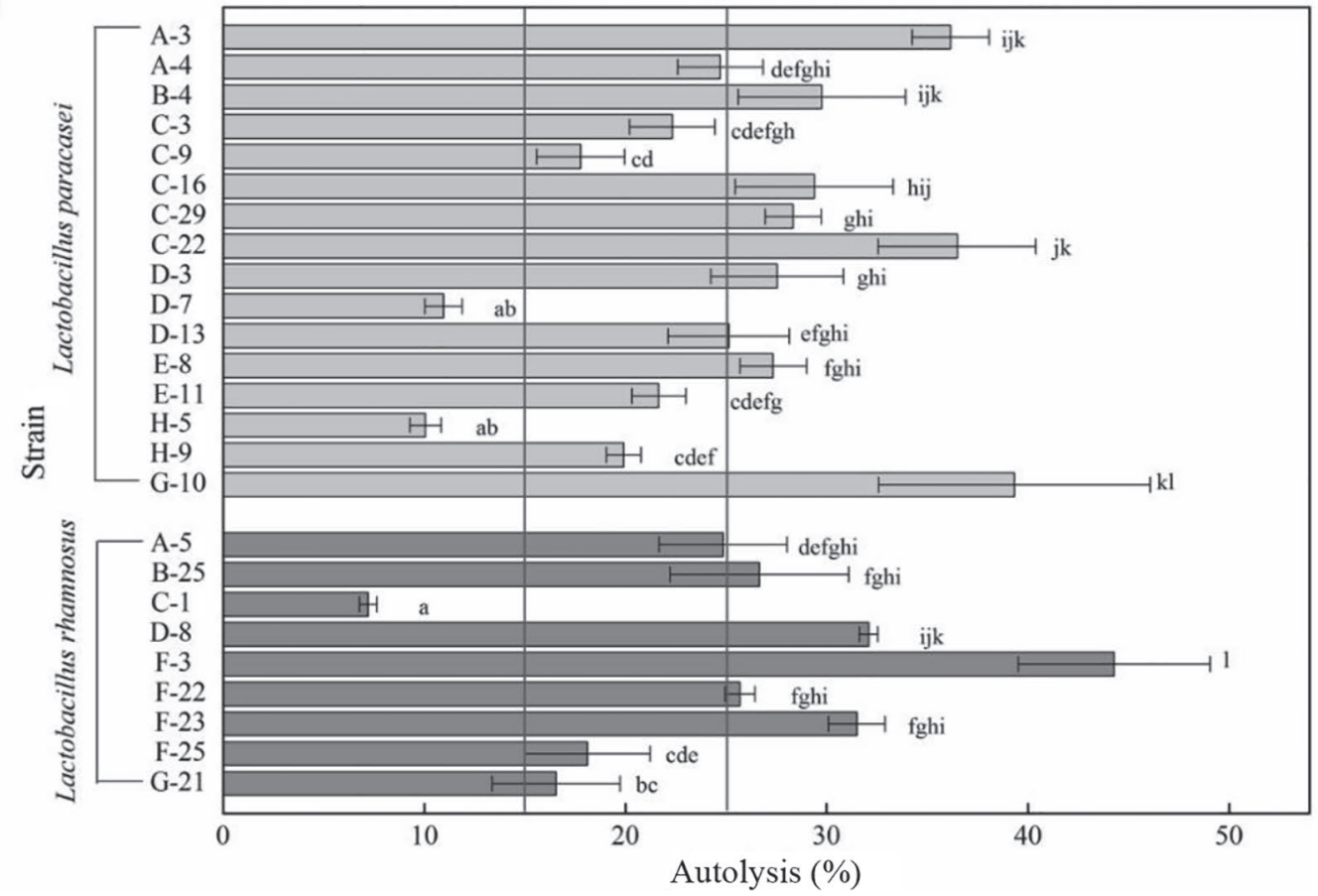

Figure 1. Acidification (a) and autolysis (b) activities of the selected lactic acid bacteria from semihard goat cheese samples (as identified in Table 1). Bars sharing the same letter $(\mathrm{a}-1)$ show no significant difference according to LSD test, $P<0.05$.

In our study, 16 strains presented a slow acidification activity, with $\Delta \mathrm{pH} \leq 0.4 \mathrm{U}$ after $6 \mathrm{~h}$ according to the classification suggested by Ayad et al. (2004). Both $L b$. paracasei and $L b$. rhamnosus strains exhibited slow acidification activities. A previous study showed that
Lb. paracasei isolated from Tenerife cheese possessed low acidification activity (Pérez et al., 2003). Similar results were expected because lactobacilli are known to slowly metabolize lactose (Carafa et al., 2015). In general, optimal NSLAB are characterized by slow to 
moderate acidification activity (Scatassa et al., 2015); high acidification activity is not favorable for NSLAB, as it would generate sensory defects in cheese.

Autolysis with the subsequent release of intracellular enzymes is a desirable trait during cheese ripening because it enhances sensory and textural properties. On the basis of the classification reported by Ayad et al. (2004), 56\% strains isolated from our cheese samples were rated good (autolysis values ranging between 25 and $65 \%$ ), $32 \%$ were rated fair (autolysis values ranging between 15 and 24\%), and the remainder (below 14\%) were rated poor (Figure 1b). The levels of autolysis for $L b$. paracase $i$ isolates ranged from 10.05 to $39.33 \%$, with the strain G-10 showing the highest $(P<0.05)$ value, whereas those of $L b$. rhamnosus isolates ranged between 7.2 and $44.28 \%$, with F-3 showing the highest value. No substantial differences were observed between the 2 species. High autolytic activities, which may accelerate cheese flavor development, affect the proteolysis rate and may reduce the bitterness of cheese by hydrolyzing large hydrophobic peptides (Nieto-Arribas et al., 2010). In this respect, our isolates could be considered as interesting NSLAB candidates for cheese manufacture.

\section{Proteolytic and Lipolytic Activities}

Cheese quality is closely related to the proteolytic activity of the bacteria present in cheese. During milk fermentation, proteolytic enzymes produced by various LAB generate biologically active peptides and AA, which contribute to the development of the flavor and texture of the dairy product. The proteases from both $L b$. paracasei and Lb. rhamnosus are less inhibited by cheese conditions, such as the presence of salt and moderately low $\mathrm{pH}$, than proteinases of Lactococcus lactis (Gobbetti and Minervini, 2014). The results shown in Table 1 indicate that $9 \mathrm{Lb}$. paracasei and $6 \mathrm{Lb}$. rhamnosus strains possessed proteolytic activity. Genome analysis also showed that NSLAB encode a relatively high number of proteolytic system components, such as proteases, peptide transporters, and peptidases. In addition, Pérez et al. (2003) observed that $85.7 \%$ $(18 / 21)$ Lb. paracasei from Tenerife cheese possessed proteolytic ability, whereas we observed a ratio of $100 \%$ $(1 / 1)$ in our study. The isolate E-11 that presented the highest acidification activity did not present an obvious proteolytic activity; this result was also reported by other studies (Nieto-Arribas et al., 2009; Fguiri et al., 2016). Proteolysis could also contribute to the prevention of allergies observed in children under $3 \mathrm{yr}$ of age due to the poor digestibility of milk proteins. However, it is noteworthy that high proteolytic activity is not always the most desired characteristic for a strain to be used in a nonstarter culture. As a matter of fact, excessive proteolysis can cause uncontrolled production of bitter peptides and other undesirable compounds, or even excessive casein hydrolysis, which would result in an excessively soft final product (Bonomo and Salzano, 2013).

None of the isolates showed lipolytic activity when assayed on tributyrin agar (Table 1). Our results corroborate those obtained by Nieto-Arribas et al. (2009), who used cream fat agar for the assay; Monfredini et al. (2012), who assayed on tributyrin agar; and Carafa et al. (2015), who used plate count agar plus milk cream for the assay. Similar results were also observed for $L b$. rhamnosus from Tenerife cheese (Pérez et al., 2003). Although LAB generally possesses weak lipolytic activity, the high concentration of LAB over an extended ripening period resulted in the generation of significant levels of free fatty acids, which are precursors of volatile aroma compounds; therefore, low lipolytic activity is considered to be an important advantageous trait for cheese production (Nieto-Arribas et al., 2010). Excessive lipolysis may generate a bitter and rancid taste in cheese (Monfredini et al., 2012). Gobbetti et al. (2015) stated that, compared with primary starters, the genomes of NSLAB possess higher number of genes related to free fatty acid catabolism. This characteristic is highly desired for a performant NSLAB in cheese ripening.

For selecting the most promising NSLAB candidates, the results of the different tests performed have to be considered. A nonstarter culture should combine slow acidification activity with a high autolytic capacity. In our study, only 10 strains with low acidification activity and acceptable autolysis values were identified. Upon considering other test results, 8 strains (A-3, B-4, D-3, E-8, G-10, B-25, D-8, and F-23) presented strong $\mathrm{NaCl}$ tolerance, along with adequate proteolytic ability and EPS- and diacetyl-producing capabilities. Overall, these strains are promising NSLAB candidates for cheese manufacture and were thus selected for subsequent examination.

\section{Aminopeptidase, Dipeptidyl Aminopeptidase, and Dipeptidase Activities}

The peptidase activity of the selected LAB strains was determined to further evaluate their potential for use as NSLAB. During secondary proteolysis, the peptidolytic potential increases the amount of small peptides and free $\mathrm{AA}$, the major precursors of specific flavor compounds. Aminopeptidases play an important role in the hydrolysis of bitter peptides and in AA liberation. Our results indicated that all strains showed an aminopeptidase activity toward the substrates Lys- $\rho$ NA, Leu- $\rho$ NA, and Pro- $\rho$ NA (Figure 2a). Similarly, these 
activities were also observed by Morea et al. (2007) in some strains of $L b$. paracasei. In addition, our results were in agreement with those of Carafa et al. (2015), who indicated that $L b$. paracasei and Lb. rhamnosus from the traditional mountain cheese Malga also exhibited activities toward Lys- $\rho$ NA and Leu- $\rho$ NA. However, Pérez et al. (2003), who studied the aminopeptidase activity of isolates from Tenerife cheese, observed that Lb. paracasei did not hydrolyze Pro- $\rho \mathrm{NA}$; this may be because the activity was strain-dependent. In addition, previous studies showed that $L b$. paracasei possessed the highest aminopeptidase activities compared with leuconostoc, lactococcal, or enterococcal strains commonly isolated from cheese (Ayad et al., 2004; González et al., 2010). In our study, D-3 (Lb. paracasei) showed the highest activity toward Leu- $\rho \mathrm{NA}$, and D-8 ( $L b$. rhamnosus) exhibited the highest activity toward Lys$\rho \mathrm{NA}$ and Pro- $\rho \mathrm{NA}$. We observed that $3 \mathrm{Lb}$. paracasei strains (B-4, D-3, and E-8) presented higher Leu- $\rho \mathrm{NA}$ activity than Lys- $\rho \mathrm{NA}$ activity, whereas others had higher Lys- $\rho \mathrm{NA}$ values than those of Leu- $\rho \mathrm{NA}$. It is noteworthy that these situations have been previously reported (Macedo et al., 2000; Bonomo and Salzano, 2013). The strong Pro- $\rho$ NA activities of our isolated LAB supported the hypothesis that the selected strains are of potential interest for use as NSLAB because they would release large amounts of proline from caseins during cheese ripening (Tsafrakidou et al., 2016). In contrast, the isolated strains E-8, B-25, and F-23 showed the lowest Lys- $\rho \mathrm{NA}$, Leu- $\rho \mathrm{NA}$, and Pro- $\rho \mathrm{NA}$ activities, respectively. Converging with our results, Nieto-Arribas et al. (2009) reported that 29 of 30 strains exhibited higher activity toward Leu- $\rho$ NA than toward Lys- $\rho$ NA, whereas Macedo et al. (2000) reported higher activity toward Lys- $\rho$ NA than Leu- $\rho$ NA for the ESB230, ESB136, and ESB117 strains.

In addition to aminopeptidase, dipeptidyl aminopeptidase specifically removes proline residues from proteins (Stefanovic et al., 2017). All of the selected strains showed dipeptidyl aminopeptidase activity toward GlyPro- $\rho$ NA and Arg-Pro- $\rho$ NA (Figure 2b). Macedo et al. (2000) obtained the same result, indicating that $L b$. paracase $i$ hydrolyzes Gly-Pro- $\rho \mathrm{NA}$ and Arg-Pro- $\rho$ NA. Reports show that $L b$. paracasei isolated from Tenerife cheese was able to hydrolyze Arg-Pro- $\rho$ NA (Pérez et al., 2003). Moreover, Lb. rhamnosus also possesses a proteolytic system including the X-prolyl-dipeptidyl aminopeptidase (Moslehishad et al., 2013). The strain D-8 (Lb. rhamnosus) exhibited the highest activity toward both Gly-Pro- $\rho$ NA and Arg-Pro- $\rho$ NA.

Our results show that each strain of the selected LAB exhibited a different dipeptidase activity (Figure 2C). Notably, the Leu-Leu peptidase activity of all strains was higher compared with those observed with other
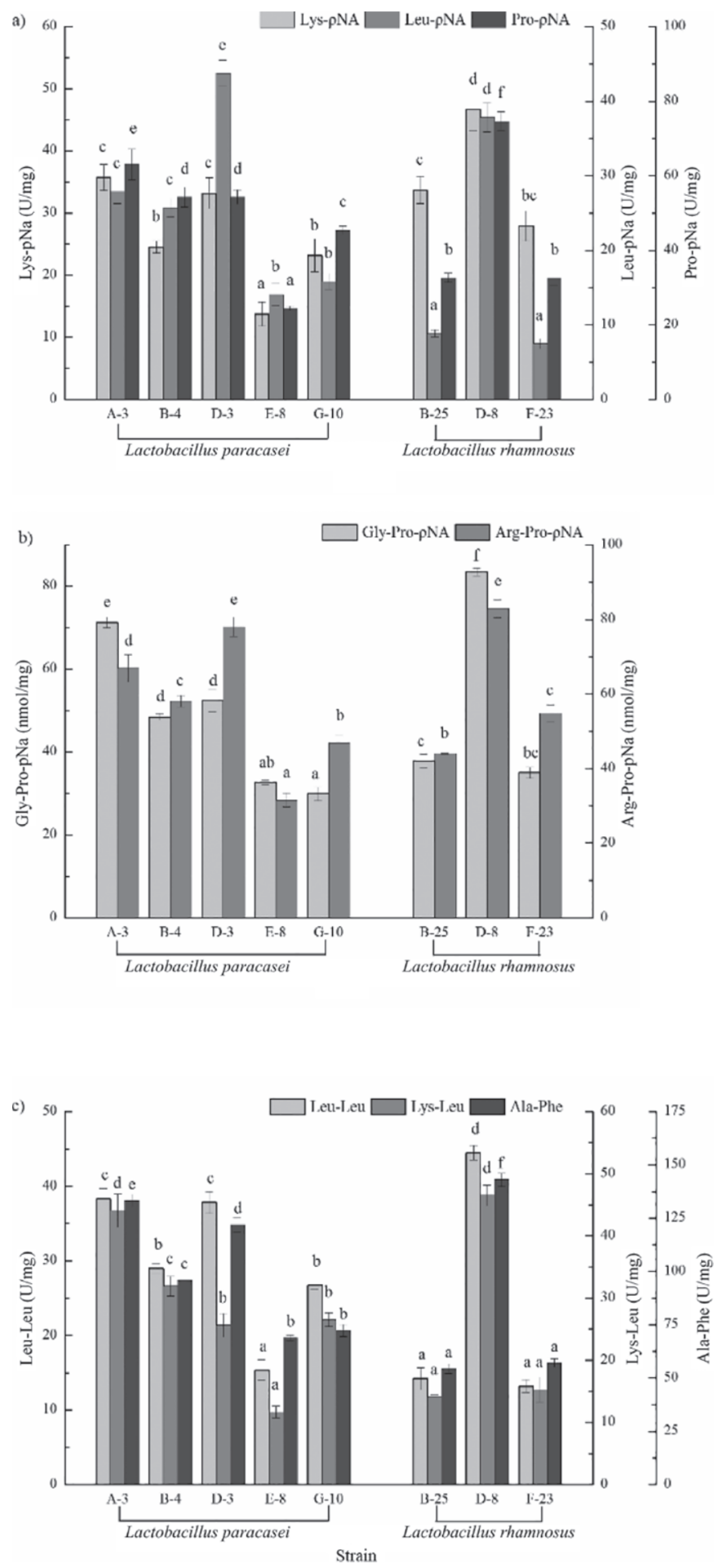

Figure 2. Peptidase activity of the selected lactic acid bacteria isolates from semihard goat cheese samples (as identified in Table 1). (a) Aminopeptidase activity is expressed as AP units, which correspond to an increase of 0.001 unit of the absorbance signal in $1 \mathrm{~min}$ for each milligram of protein. (b) Dipeptidyl aminopeptidase activity is expressed in nanomoles of nitroaniline released per milligram of protein per minute. (c) Dipeptidase activity was expressed as DP units, which correspond to an increase of 0.1 unit of the absorbance signal per minute per milligram of protein. $\rho \mathrm{NA}=\rho$-nitroanilide. 
substrates. Our results are in accordance with that reported by González et al. (2010). Highest dipeptidase activity was observed for the isolate D-8 ( Lb. rhamnosus). In view of the peptidase activity of these selected strains, A-3, A-4, D-3, and D-8 may be of interest, owing to their strong action.

The substrate specificity and extent of the peptidase activities are strain-dependent. Jensen and Ardö (2010) demonstrated that the growth medium may play a significant role in the peptidase activities of lactobacilli. The combination of proteases and peptidases permits the release of free AA, and the protease activity is generally weaker than the peptidase activity (Gobbetti and Minervini, 2014). During long ripening times of cheeses, free AA are catabolized into chemical compounds (e.g., aldehydes, ketones, alcohols, low-molecular weight sulfur compounds), which, in turn, markedly affect the sensory properties of cheese (Gobbetti and Minervini, 2014). The joint activity of peptidases and proteases is critical to achieve the desired level of proteolysis in cheeses.

\section{CONCLUSIONS}

Artisanal goat cheeses harbor diverse LAB with different properties. Our study successfully isolated, identified, and characterized the LAB from different raw goat milk cheeses for selecting potential nonstarter LAB strains. The results indicated that LAB belonging to either $L b$. paracasei or $L b$. rhamnosus showed maximum promise for manufacturing goat cheese. On the basis of the characterization of the isolated strains, 8 strains presented an acceptable resistance to high salt concentration, EPS and diacetyl production capacities, high acidification activity and autolysis, proteolytic ability, and nonlipolytic activity, and were therefore selected for further peptidase activity assay. The selected strains showed aminopeptidase, dipeptidyl aminopeptidase, and dipeptidase activities, although their hydrolysis capacities varied. Finally, 4 strains (A-3, B-4, D-3, and D-8) showing the best characteristics could be considered as NSLAB for industrial manufacturing of goat cheese.

\section{ACKNOWLEDGMENTS}

This work was financially supported by the National Natural Science Foundation of China (no. 31571850, no. 31771988), Harbin excellent subject leader project of Heilongjiang Province, China (2014RFXXJ026) and processed cheese development program of Heilongjiang Feihe Dairy Co. Ltd. (Heilongjiang Province, China; FH-YF20170620).

\section{REFERENCES}

Ayad, E. H. E., S. Nashat, N. El-Sadek, H. Metwaly, and M. El-Soda. 2004. Selection of wild lactic acid bacteria isolated from traditional Egyptian dairy products according to production and technological criteria. Food Microbiol. 21:715-725.

Bonomo, M. G., and G. Salzano. 2013. Genotypic and technological diversity of Leuconostoc mesenteroides and Lactobacillus paracasei ssp. paracasei strains for use as adjunct starter cultures in Pecorino di Filiano cheese. Int. J. Dairy Technol. 66:402-409.

Bozoudi, D., S. Torriani, A. Zdragas, and E. Litopoulou-Tzanetaki. 2016. Assessment of microbial diversity of the dominant microbiota in fresh and mature PDO Feta cheese made at three mountainous areas of Greece. Lebensm. Wiss. Technol. 72:525-533.

Briggiler-Marcó, M., M. L. Capra, A. Quiberoni, G. Vinderola, J. A. Reinheimer, and E. Hynes. 2007. Nonstarter Lactobacillus strains as adjunct cultures for cheese making: in vitro characterization and performance in two model cheeses. J. Dairy Sci. 90:4532-4542.

Burns, P., F. Cuffia, M. Milesi, G. Vinderola, C. Meinardi, N. Sabbag, and E. Hynes. 2012. Technological and probiotic role of adjunct cultures of non-starter lactobacilli in soft cheeses. Food Microbiol. $30: 45-50$.

Carafa, I., T. Nardin, R. Larcher, R. Viola, K. Tuohy, and E. Franciosi. 2015. Identification and characterization of wild lactobacilli and pediococci from spontaneously fermented mountain cheese. Food Microbiol. 48:123-132

de Almeida Júnior, W. L. G., Í. S. Ferrari, J. V. de Souza, C. D. A. da Silva, M. M. da Costa, and F. S. Dias. 2015. Characterization and evaluation of lactic acid bacteria isolated from goat milk. Food Control 53:96-103.

Di Cagno, R., I. De Pasquale, M. De Angelis, and M. Gobbetti. 2012. Accelerated ripening of Caciocavallo Pugliese cheese with attenuated adjuncts of selected nonstarter lactobacilli. J. Dairy Sci. 95:4784-4795.

Di Grigoli, A., N. Francesca, R. Gaglio, V. Guarrasi, M. Moschetti, M. L. Scatassa, L. Settanni, and A. Bonanno. 2015. The influence of the wooden equipment employed for cheese manufacture on the characteristics of a traditional stretched cheese during ripening. Food Microbiol. 46:81-91.

Domingos-Lopes, M. F., C. Stanton, P. R. Ross, M. L. Dapkevicius, and C. C. Silva. 2017. Genetic diversity, safety and technological characterization of lactic acid bacteria isolated from artisanal Pico cheese. Food Microbiol. 63:178-190.

Ercan, D., F. Korel, and H. Orşahin. 2014. Microbiological quality of artisanal Sepet cheese. Int. J. Dairy Technol. 67:384-393.

Ferrari, I. S., J. V. de Souza, C. L. Ramos, M. M. da Costa, R. F. Schwan, and F. S. Dias. 2016. Selection of autochthonous lactic acid bacteria from goat dairies and their addition to evaluate the inhibition of Salmonella typhi in artisanal cheese. Food Microbiol. 60:29-38.

Fguiri, I., M. Ziadi, M. Atigui, N. Ayeb, S. Arroum, M. Assadi, and T. Khorchani. 2016. Isolation and characterisation of lactic acid bacteria strains from raw camel milk for potential use in the production of fermented Tunisian dairy products. Int. J. Dairy Technol. 69:103-113.

Franciosi, E., L. Settanni, A. Cavazza, and E. Poznanski. 2009. Biodiversity and technological potential of wild lactic acid bacteria from raw cows' milk. Int. Dairy J. 19:3-11.

Fröhlich-Wyder, M.-T., D. Guggisberg, R. Badertscher, D. Wechsler, A. Wittwer, and S. Irmler. 2013. The effect of Lactobacillus buchneri and Lactobacillus parabuchneri on the eye formation of semihard cheese. Int. Dairy J. 33:120-128.

Gatti, M., B. Bottari, C. Lazzi, E. Neviani, and G. Mucchetti. 2014 Invited review: Microbial evolution in raw-milk, long-ripened cheeses produced using undefined natural whey starters. J. Dairy Sci. 97:573-591.

Georgieva, R., I. Iliev, T. Haertlé, J.-M. Chobert, I. Ivanova, and S. Danova. 2009. Technological properties of candidate probiotic Lactobacillus plantarum strains. Int. Dairy J. 19:696-702.

Gobbetti, M., M. De Angelis, R. Di Cagno, L. Mancini, and P. F. Fox. 2015. Pros and cons for using non-starter lactic acid bacte- 
ria (NSLAB) as secondary/adjunct starters for cheese ripening. Trends Food Sci. Technol. 45:167-178.

Gobbetti, M., and F. Minervini. 2014. Lactobacillus| Lactobacillus casei A2. Pages 432-438 in Encyclopedia of Food Microbiology. 2nd ed. M. L. Tortorello, ed. Academic Press, Oxford, UK.

González, L., N. Sacristan, R. Arenas, J. M. Fresno, and M. Eugenia Tornadijo. 2010. Enzymatic activity of lactic acid bacteria (with antimicrobial properties) isolated from a traditional Spanish cheese. Food Microbiol. 27:592-597.

Guidone, A., T. Zotta, R. P. Ross, C. Stanton, M. C. Rea, E. Parente, and A. Ricciardi. 2014. Functional properties of Lactobacillus plantarum strains: A multivariate screening study. Lebensm. Wiss. Technol. 56:69-76.

Hernández, D., E. Cardell, and V. Zarate. 2005. Antimicrobial activity of lactic acid bacteria isolated from Tenerife cheese: Initial characterization of plantaricin TF711, a bacteriocin-like substance produced by Lactobacillus plantarum TF711. J. Appl. Microbiol. 99:77-84

Herreros, M. A., J. M. Fresno, M. J. González Prieto, and M. E. Tornadijo. 2003. Technological characterization of lactic acid bacteria isolated from Armada cheese (a Spanish goats' milk cheese). Int. Dairy J. 13:469-479.

Jensen, M. P., and Y. Ardö. 2010. Variation in aminopeptidase and aminotransferase activities of six cheese related Lactobacillus helveticus strains. Int. Dairy J. 20:149-155.

Juan, B., A. Zamora, J. M. Quevedo, and A.-J. Trujillo. 2016. Proteolysis of cheese made from goat milk treated by ultra high pressure homogenisation. Lebensm. Wiss. Technol. 69:17-23.

Lazzi, C., M. Povolo, F. Locci, V. Bernini, E. Neviani, and M. Gatti. 2016. Can the development and autolysis of lactic acid bacteria influence the cheese volatile fraction? The case of Grana Padano. Int. J. Food Microbiol. 233:20-28.

Litopoulou-Tzanetaki, E., and N. Tzanetakis. 2011. Microbiological characteristics of Greek traditional cheeses. Small Rumin. Res. $101: 17-32$

Macedo, A. C., M. Vieira, R. Poças, and F. X. Malcata. 2000. Peptide hydrolase system of lactic acid bacteria isolated from Serra da Estrela cheese. Int. Dairy J. 10:769-774.

Martín-Platero, A. M., M. Maqueda, E. Valdivia, J. Purswani, and M. Martinez-Bueno. 2009. Polyphasic study of microbial communities of two Spanish farmhouse goats' milk cheeses from Sierra de Aracena. Food Microbiol. 26:294-304.

Martínez, S., I. Franco, and J. Carballo. 2011. Spanish goat and sheep milk cheeses. Small Rumin. Res. 101:41-54.

Monfredini, L., L. Settanni, E. Poznanski, A. Cavazza, and E. Franciosi. 2012. The spatial distribution of bacteria in Grana-cheese during ripening. Syst. Appl. Microbiol. 35:54-63.

Morea, M., A. Matarante, R. Di Cagno, F. Baruzzi, and F. Minervini. 2007. Contribution of autochthonous non-starter lactobacilli to proteolysis in Caciocavallo Pugliese cheese. Int. Dairy J. 17:525534

Moslehishad, M., M. R. Ehsani, M. Salami, S. Mirdamadi, H. Ezzatpanah, A. N. Naslaji, and A. A. Moosavi-Movahedi. 2013. The comparative assessment of ACE-inhibitory and antioxidant activities of peptide fractions obtained from fermented camel and bovine milk by Lactobacillus rhamnosus PTCC 1637. Int. Dairy J. 29:82-87.

Navarro-Alarcón, M., C. Cabrera-Vique, M. D. Ruiz-López, M. Olalla, R. Artacho, R. Giménez, V. Quintana, and T. Bergillos. 2011. Levels of $\mathrm{Se}, \mathrm{Zn}, \mathrm{Mg}$ and $\mathrm{Ca}$ in commercial goat and cow milk fermented products: Relationship with their chemical composition and probiotic starter culture. Food Chem. 129:1126-1131.

Nieto-Arribas, P., J. M. Poveda, S. Seseña, L. Palop, and L. Cabezas. 2009. Technological characterization of Lactobacillus isolates from traditional Manchego cheese for potential use as adjunct starter cultures. Food Control 20:1092-1098.

Nieto-Arribas, P., S. Sesena, J. M. Poveda, L. Palop, and L. Cabezas. 2010. Genotypic and technological characterization of Leuconostoc isolates to be used as adjunct starters in Manchego cheese manufacture. Food Microbiol. 27:85-93.

Nikolic, M. A. Terzic-Vidojevic, B. Jovcic, J. Begovic, N. Golic, and L. Topisirovic. 2008. Characterization of lactic acid bacteria isolated from Bukuljac, a homemade goat's milk cheese. Int. J. Food Microbiol. 122:162-170.

Pacheco Da Silva, F. F., V. Biscola, J. G. LeBlanc, and B. D. Gombossy de Melo Franco. 2016. Effect of indigenous lactic acid bacteria isolated from goat milk and cheeses on folate and riboflavin content of fermented goat milk. Lebensm. Wiss. Technol. 71:155161.

Pérez, G., E. Cardell, and V. Zárate. 2003. Technological characterization of lactic acid bacteria from Tenerife cheese. Int. J. Food Sci Technol. 38:537-546.

Piras, C., F. Cesare Marincola, F. Savorani, S. B. Engelsen, S. Cosentino, S. Viale, and M. B. Pisano. 2013. A NMR metabolomics study of the ripening process of the Fiore Sardo cheese produced with autochthonous adjunct cultures. Food Chem. 141:2137-2147.

Ribeiro, S. C., M. C. Coelho, S. D. Todorov, B. D. Franco, M. L. Dapkevicius, and C. C. Silva. 2014. Technological properties of bacteriocin-producing lactic acid bacteria isolated from Pico cheese an artisanal cow's milk cheese. J. Appl. Microbiol. 116:573-585.

Sánchez, I., S. Sesena, J. M. Poveda, L. Cabezas, and L. Palop. 2005. Phenotypic and genotypic characterization of lactobacilli isolated from Spanish goat cheeses. Int. J. Food Microbiol. 102:355-362.

Scatassa, M. L., R. Gaglio, G. Macaluso, N. Francesca, W. Randazzo, C. Cardamone, A. Di Grigoli, G. Moschetti, and L. Settanni. 2015. Transfer, composition and technological characterization of the lactic acid bacterial populations of the wooden vats used to produce traditional stretched cheeses. Food Microbiol. 52:31-41.

Serhan, M., M. Linder, C. Hosri, and J. Fanni. 2010. Changes in proteolysis and volatile fraction during ripening of Darfiyeh, a Lebanese artisanal raw goat's milk cheese. Small Rumin. Res. 90:75-82.

Stefanovic, E., K. N. Kilcawley, M. C. Rea, G. F. Fitzgerald, and O. McAuliffe. 2017. Genetic, enzymatic and metabolite profiling of the Lactobacillus casei group reveals strain biodiversity and potential applications for flavour diversification. J. Appl. Microbiol. 122:1245-1261.

Terzic-Vidojevic, A., S. Mihajlovic, G. Uzelac, K. Veljovic, M. Tolinacki, M. Nikolic, L. Topisirovic, and M. Kojic. 2014. Characterization of lactic acid bacteria isolated from artisanal Travnik young cheeses, sweet creams and sweet kajmaks over four seasons. Food Microbiol. 39:27-38

Thierry, A., F. Valence, S.-M. Deutsch, S. Even, H. Falentin, Y. Le Loir, G. Jan, and V. Gagnaire. 2015. Strain-to-strain differences within lactic and propionic acid bacteria species strongly impact the properties of cheese-A review. Dairy Sci. Technol. 95:895-918.

Tsafrakidou, P., D. Bozoudi, S. Pavlidou, C. Kotzamanidis, M. Hatzikamari, A. Zdragas, and E. Litopoulou-Tzanetaki. 2016. Technological, phenotypic and genotypic characterization of lactobacilli from Graviera Kritis PDO Greek cheese, manufactured at two traditional dairies. Lebensm. Wiss. Technol. 68:681-689. 